\title{
Perspectives on the Barrier to Resistance for Dolutegravir + Lamivudine, a Two-Drug Antiretroviral Therapy for HIV-1 Infection
}

\author{
Marta Boffito, Laura Waters, ${ }^{2}$ Pedro Cahn, ${ }^{3}$ Roger Paredes, ${ }^{4}$ Justin Koteff, Jean Van Wyk, ${ }^{6}$ Tia Vincent, ${ }^{6}$ \\ James Demarest, ${ }^{5}$ Kimberly Adkison, ${ }^{6}$ and Romina Quercia ${ }^{6}$
}

\begin{abstract}
In HIV-1-infected patients, virological failure can occur as a consequence of the mutations that accumulate in the viral genome that allow replication to continue in the presence of antiretrovirals (ARVs). The development of treatment-emergent resistance to an ARV can limit a patient's options for future therapy, prompting the need for ARV regimens that are resilient to the emergence of resistance. The genetic barrier to resistance refers to the number of mutations in an ARV's therapeutic target that are required to confer a clinically meaningful loss of susceptibility to the drug. The emergence of resistance can be affected by pharmacological aspects of the ARV, including its structure, inhibitory quotient, therapeutic index, and pharmacokinetic characteristics. Dolutegravir (DTG) has demonstrated a high barrier to resistance, including when used in a two-drug regimen (2DR) with lamivudine (3TC). In the GEMINI1 and GEMINI-2 studies, DTG +3TC was noninferior to DTG + emtricitabine/tenofovir disoproxil fumarate in treatment-naive participants, with similar proportions achieving HIV-1 RNA $<50$ copies $/ \mathrm{mL}$ through 96 weeks. Furthermore, in the TANGO study, virological suppression was maintained at 48 weeks after switching to DTG +3TC from a tenofovir alafenamide (TAF)-based regimen compared with continuing a TAF-based regimen. Most other 2DRs with successful outcomes compared with three-drug regimens have been based on protease inhibitors (PIs); however, this class is associated with adverse metabolic effects and drug-drug interactions. In this review, we discuss the barrier to resistance in the context of a 2DR in which a boosted PI is replaced with DTG +3TC.
\end{abstract}

Keywords: HIV, antiretroviral, genetic barrier to resistance, two-drug regimen, integrase strand transfer inhibitor

\section{Introduction}

$\mathbf{V}$

IROLOGICAL FAILURE IN HIV-1-infected patients can be associated with emergence of antiretroviral (ARV) drug resistance, narrowing options for future therapy. ${ }^{1}$ Although the genetic barrier to resistance describes the threshold of mutations required for clinically meaningful loss of drug susceptibility, ${ }^{2}$ the emergence of resistance can also be influenced by the drug's structure, ${ }^{3}$ inhibitory quotient, ${ }^{4}$ therapeutic index ${ }^{5}$ and pharmacokinetic forgiveness. ${ }^{6}$

Several two-drug regimens (2DRs) have been compared with three-drug regimens (3DRs) in clinical studies, most of which examined regimens based on boosted protease inhibitors (PIs) ${ }^{7-9}$ Although PIs demonstrate a high barrier to resistance, ${ }^{10-12}$ they are associated with metabolic adverse effects and substantial drug-drug interactions compared with other ARV classes. ${ }^{13,14}$ Second-generation integrase strand transfer inhibitors (INSTIs) offer the possibility of non-PIbased 2DRs. A 2DR based on the INSTI dolutegravir (DTG) demonstrated noninferiority to a 3DR in a treatment-naive population in the GEMINI-1 and GEMINI-2 studies through 48 weeks, as well as no selection of resistance mutations among those with confirmed virological withdrawal. ${ }^{15} \mathrm{Im}$ portantly, similar proportions of participants achieved HIV-1

\footnotetext{
${ }^{1}$ Chelsea and Westminister Hospital, London, United Kingdom.

${ }^{2}$ Mortimer Market Centre, London, United Kingdom.

${ }^{3}$ Fundación Huésped, Buenos Aires, Argentina.

${ }^{4}$ Hospital Germans Trias i Pujol, Catalonia, Spain.

${ }^{5}$ ViiV Healthcare, Research Triangle Park, North Carolina.

${ }^{6}$ ViiV Healthcare, Brentford, United Kingdom.
}

(c) Marta Boffito et al. 2019; Published by Mary Ann Liebert, Inc. This Open Access article is distributed under the terms of the Creative Commons License (http://creativecommons.org/licenses/by/4.0), which permits unrestricted use, distribution, and reproduction in any medium, provided the original work is properly cited. 
RNA $<50$ copies/mL through 96 weeks in both study groups, with no further emergence of resistance. ${ }^{16}$ Furthermore, in the TANGO study, virological suppression was maintained for 48 weeks in a treatment-experienced population after switching from a tenofovir alafenamide (TAF)-based regimen to DTG + lamivudine (3TC), with no observation of confirmed virological withdrawal or treatment-emergent resistance in the DTG +3TC group. ${ }^{17}$ We discuss the barrier to resistance concept in the context of the $2 \mathrm{DR}$ DTG $+3 \mathrm{TC}$.

\section{Barrier to Resistance}

ARV development focuses on optimizing new agents that are resilient to developing resistance and retain activity in the presence of existing resistance-associated mutations. Secondgeneration INSTIs [e.g., DTG, bictegravir (BIC)] present improved resistance profiles compared with first-generation INSTIs [e.g., raltegravir (RAL), elvitegravir (EVG)]. ${ }^{18-21}$

In clinical trials that compared DTG and other ARVs combined with two nucleoside reverse transcriptase inhibitors (NRTIs) in treatment-naive participants, no treatmentemergent DTG resistance has been observed. ${ }^{18,22-25}$ Among participants in the SPRING-2 study with virological failure and available genotypic results, INSTI resistance was not observed in the DTG group $(n=8)$ but was detected in $1(6 \%)$ of 18 in the RAL group; none of 12 in the DTG group and 4 $(21 \%)$ of 19 in the RAL group developed NRTI resistance. ${ }^{18}$ Two studies comparing BIC- and DTG-based 3DRs reported noninferior efficacy and no treatment-emergent resistance in either group. $^{24,25}$ The FLAMINGO and ARIA studies compared DTG and boosted PIs in treatment-naive participants with no INSTI or PI resistance observed. ${ }^{22,23}$

\section{Pharmacological Aspects}

\section{Drug structure}

The interaction between an ARV and its target at the structural level influences emergence of HIV-1 resistance. Although INSTIs have a conserved mode of binding to viral integrase ${ }^{26}$ resistance mutations affect interactions between each INSTI and its binding site differently, creating varied effects on ARV activity. ${ }^{3}$ In a study comparing structural and functional characteristics of DTG, RAL, and EVG, DTG was shown to extend farther into the binding site and have more flexibility to adjust its position in the presence of amino acid substitutions than RAL and EVG. ${ }^{3}$ These characteristics are attributed to the length and flexibility of the linker connecting the tricyclic metal-chelating core and the difluorophenyl ring and may allow DTG to retain antiviral activity in the presence of mutations that confer resistance to other INSTIs. ${ }^{3}$

DTG exhibits a longer half-life of dissociation from wildtype integrase-DNA complex $(71 \mathrm{~h})$ compared with RAL $(8.8 \mathrm{~h})$ and $\operatorname{EVG}(2.7 \mathrm{~h}),{ }^{27}$ and a dissociation half-life of $135 \mathrm{~h}$ has been reported for $\mathrm{BIC} .^{28}$ Prolonged residence time at the active site may extend the duration of efficacy, contributing to a higher barrier to resistance. ${ }^{27}$

\section{Inhibitory quotient}

The inhibitory quotient expresses the potency of a drug as the ratio of drug exposure to viral susceptibility ${ }^{29}$ and is measured as the concentration of drug required to suppress replication of wild-type virus at a specified level, often $50 \%$
$\left(\mathrm{IC}_{50}\right)$ or $90 \%\left(\mathrm{IC}_{90}\right) .{ }^{10}$ DTG has an $\mathrm{IC}_{90}$ of $0.064 \mu \mathrm{g} / \mathrm{mL}$, 17-fold below plasma concentrations observed at the end of the dosing interval for a once-daily, 50-mg dose $\left(\mathrm{C}_{\text {trough }}\right.$; $1.1 \mu \mathrm{g} / \mathrm{mL}),{ }^{30}$ making DTG a potent ARV, even in the presence of mutations that reduce viral susceptibility to RAL or EVG. ${ }^{31}$

\section{Therapeutic index}

To be clinically useful, an ARV should have a wide therapeutic index, meaning viral suppression can be achieved with doses well below those that cause toxicity. ${ }^{5}$ Because ARVs are used in patients at concentrations well above those used to evaluate susceptibility in vitro, prediction of the ability of a drug to suppress viral replication at clinically relevant concentrations requires understanding the dose-response relationship. ${ }^{32}$

DTG has a wide therapeutic index; it is able to suppress viral replication at concentrations far below those shown to cause cytotoxicity. ${ }^{31}$ In a pharmacokinetic study in HIV-1infected patients, the relationship between $\mathrm{C}_{\text {trough }}$ and HIV-1 RNA decline for 10 days of DTG monotherapy could be described with a simple maximum effect $\left(E_{\max }\right)$ model with $E_{\max }=-2.6 \log _{10}$ and a $50 \%$ effective concentration of $0.036 \mu \mathrm{g} / \mathrm{mL} .{ }^{33}$ In a dose-ranging study, similar proportions of participants treated with DTG at 2, 10, and $50 \mathrm{mg}$ experienced drug-related adverse effects, which were mostly mild to moderate in severity. ${ }^{34}$ Together, these findings demonstrate that DTG is well tolerated at an effective dose, supporting a wide therapeutic index.

\section{Pharmacokinetic forgiveness}

Pharmacokinetic forgiveness is the difference between the duration of beneficial action after dosing and the prescribed dosing interval. ${ }^{35} \mathrm{ARV}$ forgiveness relates to the number of doses that can be missed without causing viral relapse. Suboptimal adherence may lead to inadequate ARV exposure, virological failure, and drug resistance. ${ }^{6,36}$ Forgiveness in the context of missed doses is possible when either the elimination half-life of a drug or its inhibitory effect exceeds the recommended dosing interval. ${ }^{6}$

DTG has a longer elimination half-life than EVG and RAL, suggesting that it may be more forgiving of missed doses. ${ }^{37}$ The terminal elimination half-life of DTG is $\sim 14 \mathrm{~h},{ }^{30}$ compared with $12.9 \mathrm{~h}$ for EVG when boosted with cobicistat $^{38}$ and 10-12 $\mathrm{h}$ for RAL. ${ }^{39}$ For BIC, the half-life reported in HIV-1-infected, treatment-, or INSTI-naive participants ranged from 16 to $21 \mathrm{~h}^{40}$

The duration of the inhibitory effect of a drug also affects forgiveness. Plasma concentrations of DTG were $>2$-fold higher than the $\mathrm{IC}_{90}$ for $72 \mathrm{~h}$ after the last dose, whereas the concentration of EVG when boosted with cobicistat only exceeded the $\mathrm{IC}_{95}$ through $36 \mathrm{~h}$, further supporting higher forgiveness of missed doses for DTG. ${ }^{37}$ Combination therapy should include drugs with complementary pharmacokinetic profiles, such as those demonstrated for DTG and the pharmacologically active triphosphate form of 3TC, which have similar half-lives that support once-daily dosing (Fig. 1). ${ }^{34,41,42}$

Synergistic interactions can occur among ARVs in combination. ${ }^{43}$ In vitro studies measuring inhibition of viral replication by DTG in combination with other ARVs found that combinations of DTG with two NRTIs [abacavir/3TC or tenofovir disoproxil fumarate/emtricitabine (TDF/FTC)] presented greater antiviral activity than expected if the effects 
FIG. 1. Steady-state DTG and intracellular 3TC-TP concentrationtime profiles after administration of DTG $50 \mathrm{mg}$ or 3TC-TP $300 \mathrm{mg}$ daily. ${ }^{34,41,42}$ BID, twice daily; conc, concentration; DTG, dolutegravir; PA- $\mathrm{IC}_{90}$, protein-adjusted $90 \%$ inhibitory concentration; QD, once daily; 3TC-TP, lamivudine triphosphate.

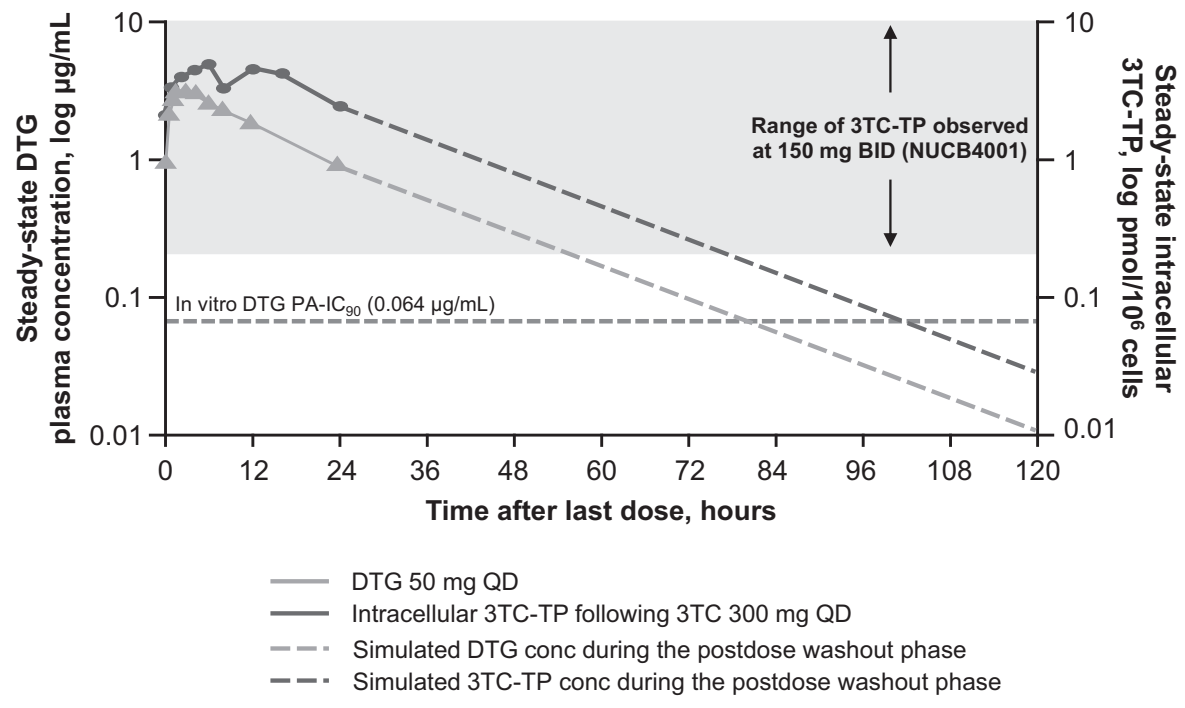

were additive. ${ }^{44}$ This conclusion is consistent with a metaanalysis of studies reporting virological failure among virologically suppressed patients switched to DTG-based 2DRs, which demonstrated that the efficacy of DTG-based combination therapies was considerably higher than that of DTG monotherapy. ${ }^{45}$ Importantly, $>50 \%$ of those experiencing virological failure on DTG monotherapy developed resistance, whereas no treatment-emergent resistance was recorded in patients who received DTG-based 2DRs. ${ }^{45}$

\section{Two-Drug Regimens}

There is considerable interest in identifying 2DRs with noninferior efficacy, improved tolerability, and reduced potential for long-term adverse events compared with 3DRs. ${ }^{46}$ 2DRs should combine agents having high potency and a high barrier to resistance; accordingly, most successful 2DRs that have been evaluated have included boosted PIs. ${ }^{7-9,47,48}$ In studies investigating 2DRs, regimens composed of a ritonavir-boosted PI +3 TC have shown similar outcomes to 3DR comparators and no or minimal resistance. ${ }^{9,47,49}$

Studies evaluating INSTI-based 2DRs include the NEAT001, PROGRESS, and SECOND-LINE studies, which all examined 2DRs composed of a ritonavir-boosted PI + RAL. ${ }^{48,50,51}$ In each of these studies, the 2DR demonstrated noninferior efficacy compared with the 3DR; however, mutations associated with RAL resistance emerged in participants who developed virological failure while receiving the 2DR.

DTG is a strong candidate for 2DRs based on phase III trials demonstrating its high barrier to resistance. ${ }^{15,18,22,23,52}$ In the GEMINI-1 and GEMINI-2 studies, virological suppression with the $2 \mathrm{DR}$ DTG $+3 \mathrm{TC}$ was noninferior to that of the 3DR DTG + TDF/FTC as first-line treatment. ${ }^{15}$ Viral suppression (HIV-1 RNA <50 copies/mL) was achieved in $91 \%(655 / 716)$ and $93 \%$ (669/717) of participants in the 2DR and 3DR groups, respectively, at week 48 . Both study groups exhibited similar rapid viral load log decline and median time to viral suppression overall and among participants with baseline viral load $>100,000$ copies $/ \mathrm{mL}$, including those with viral load $>500,000$ copies $/ \mathrm{mL}$ at baseline; high proportions of participants achieved viral suppression, irrespective of baseline viral load. ${ }^{53}$ No INSTI or NRTI resistance mutations were detected in participants with confirmed virological withdrawal at 48 weeks (2DR, $n=6 ; 3 \mathrm{DR}, n=4) .{ }^{15}$ The noninferiority of the 2DR was maintained at 96 weeks, and there was no development of resistance in either study group. ${ }^{16}$

In the TANGO study, participants with stable virological suppression for $\geq 6$ months on a TAF-based regimen were randomized to switch to DTG $+3 \mathrm{TC}$ or to continue on a TAFbased regimen. ${ }^{17}$ The primary endpoint was the proportion of participants with virological failure at 48 weeks according to the United States Food and Drug Administration Snapshot algorithm. The study successfully demonstrated the noninferiority of the 2DR to a TAF-based regimen, and there was no occurrence of confirmed virological withdrawal or emergence of resistance in the DTG +3 TC group, making this the first randomized controlled trial in a switch population to report such findings in treatment-experienced individuals.

With promising results from GEMINI- 1 and GEMINI- 2 in a treatment-naive population and from TANGO in a treatment-experienced population, we are entering an era when a high resistance barrier can be achieved by combining two optimal unboosted drugs. Nonetheless, it should be noted that although these findings are encouraging, the clinical relevance of the pharmacological aspects of resistance discussed in this review is unknown. In addition, as clinical experience with the $2 \mathrm{DR}$ of DTG $+3 \mathrm{TC}$ is limited to the clinical trial setting, the effect of recurrent nonadherence on the emergence of resistance remains to be determined. Finally, it is difficult to evaluate the barrier to resistance because of the small subset of participants who experienced confirmed virological withdrawal, although the low rate of confirmed virological withdrawal in the GEMINI studies and the absence of confirmed virological withdrawal in the TANGO study affirm the high efficacy of the regimen.

\section{Author Disclosure Statement}

M.B. has received speaking and advising fees from Gilead, ViiV Healthcare, Merck Sharp \& Dohme, and Janssen; traveling grants from Gilead; and research grants to the institution from Gilead, ViiV Healthcare, Merck Sharp \& 
Dohme, Janssen, Bristol-Myers Squibb, and Mylan. L.W. reports personal fees from ViiV Healthcare, Gilead, Merck Sharp \& Dohme, Janssen, and Mylan outside the submitted study; L.W. is a member of the HIV Clinical Reference Group, advising NHS England on HIV treatment and care; L.W. cochairs the drugs subgroup; L.W. chairs the British HIV Association ART guidelines. P.C. reports grants from AbbVie, advisory board fees from Merck Sharp \& Dohme, and grants and advisory board fees from ViiV Healthcare outside the submitted study. R.P. reports grants and consulting fees from ViiV Healthcare, Merck Sharp \& Dohme, and Gilead during the conduct of the study. J.K., J.V.W., T.V., K.A., and R.Q. are employees of ViiV Healthcare and own stock in GlaxoSmithKline. J.D. was an employee of ViiV Healthcare during the time of the study and owns stock/stock options in GlaxoSmithKline.

\section{Funding Information}

This review was funded by ViiV Healthcare. Editorial assistance was provided under the direction of the authors by Leila Strickland, PhD, and Diane Neer, ELS, MedThink SciCom.

\section{References}

1. Isaac A, Pillay D: New drugs for treating drug resistant HIV-1. Sex Transm Infect 2003;79:176-178.

2. Luber AD: Genetic barriers to resistance and impact on clinical response. MedGenMed 2005;7:69.

3. Hare S, Smith SJ, Métifiot M, et al.: Structural and functional analyses of the second-generation integrase strand transfer inhibitor dolutegravir (S/GSK1349572). Mol Pharmacol 2011;80:565-572.

4. Tang MW, Shafer RW: HIV-1 antiretroviral resistance: Scientific principles and clinical applications. Drugs 2012; 72:e1-e25.

5. US Department of Health and Human Services, Food and Drug Administration, Center for Drug Evaluation and Research (CDER): Guidance for industry: Role of HIV resistance testing in antiretroviral drug development. Available at https://fda.gov/regulatory-information/searchfda-guidance-documents/role-hiv-drug-resistance-testingantiretroviral-drug-development (2007), accessed June 24, 2019.

6. Shuter J: Forgiveness of non-adherence to HIV-1 antiretroviral therapy. J Antimicrob Chemother 2008;61:769773.

7. Riddler SA, Haubrich R, DiRienzo AG, et al.: Classsparing regimens for initial treatment of HIV-1 infection. N Engl J Med 2008;358:2095-2106.

8. Arribas JR, Girard PM, Landman R, et al.: Dual treatment with lopinavir-ritonavir plus lamivudine versus triple treatment with lopinavir-ritonavir plus lamivudine or emtricitabine and a second nucleos(t)ide reverse transcriptase inhibitor for maintenance of HIV-1 viral suppression (OLE): A randomised, open-label, non-inferiority trial. Lancet Infect Dis 2015; 15:785-792.

9. Di Giambenedetto S, Fabbiani M, Quiros Roldan E, et al.: Treatment simplification to atazanavir/ritonavir + lamivudine versus maintenance of atazanavir/ritonavir + two NRTIs in virologically suppressed HIV-1-infected patients: 48 Week results from a randomized trial (ATLAS-M). J Antimicrob Chemother 2017;72:1163-1171.
10. Clutter DS, Jordan MR, Bertagnolio S, Shafer RW: HIV-1 drug resistance and resistance testing. Infect Genet Evol 2016;46:292-307.

11. de Meyer S, Vangeneugden T, van Baelen B, et al.: Resistance profile of darunavir: Combined 24-week results from the POWER trials. AIDS Res Hum Retroviruses 2008; 24:379-388

12. Mo H, King MS, King K, Molla A, Brun S, Kempf DJ: Selection of resistance in protease inhibitor-experienced, human immunodeficiency virus type 1-infected subjects failing lopinavir- and ritonavir-based therapy: Mutation patterns and baseline correlates. J Virol 2005;79:33293338.

13. Fontas E, van Leth F, Sabin CA, et al.: Lipid profiles in HIV-infected patients receiving combination antiretroviral therapy: Are different antiretroviral drugs associated with different lipid profiles? J Infect Dis 2004;189:1056-1074.

14. Patel N, Abdelsayed S, Veve M, Miller CD: Predictors of clinically significant drug-drug interactions among patients treated with nonnucleoside reverse transcriptase inhibitor-, protease inhibitor-, and raltegravir-based antiretroviral regimens. Ann Pharmacother 2011;45:317-324.

15. Cahn P, Madero JS, Arribas JR, et al.: Dolutegravir plus lamivudine versus dolutegravir plus tenofovir disoproxil fumarate and emtricitabine in antiretroviral-naive adults with HIV-1 infection (GEMINI-1 and GEMINI-2): Week 48 results from two multicentre, double-blind, randomised, non-inferiority, phase 3 trials. Lancet 2019;393:143-155.

16. Cahn P, Sierra Madero JS, Arribas J, et al:: Durable efficacy of dolutegravir (DTG) plus lamivudine (3TC) in antiretroviral treatment-naive adults with HIV-1 infection: 96-Week results from the GEMINI studies. Abstract presented at: 10th IAS Conference on HIV Science; July 2124, 2019. Mexico City, Mexico.

17. van Wyk J, Ajana F, Bisshop F, et al: Switching to DTG/3TC fixed-dose combination (FDC) is non-inferior to continuing a TAF-based regimen in maintaining virologic suppression through 48 weeks (TANGO study). Abstract presented at: 10th IAS Conference on HIV Science; July 21-24, 2019. Mexico City, Mexico.

18. Raffi F, Rachlis A, Stellbrink HJ, et al.: Once-daily dolutegravir versus raltegravir in antiretroviral-naive adults with HIV-1 infection: 48 Week results from the randomised, double-blind, non-inferiority SPRING-2 study. Lancet 2013;381:735-743.

19. Cahn P, Pozniak AL, Mingrone $\mathrm{H}$, et al.: Dolutegravir versus raltegravir in antiretroviral-experienced, integraseinhibitor-naive adults with HIV: Week 48 results from the randomised, double-blind, non-inferiority SAILING study. Lancet 2013;382;700-708.

20. Akil B, Blick G, Hagins DP, et al:: Dolutegravir versus placebo in subjects harbouring HIV-1 with integrase inhibitor resistance associated substitutions: 48-Week results from VIKING-4, a randomized study. Antivir Ther 2015; 20:343-348.

21. Castagna A, Maggiolo F, Penco G, et al.: Dolutegravir in antiretroviral-experienced patients with raltegravir- and/or elvitegravir-resistant HIV-1: 24-Week results of the phase III VIKING-3 study. J Infect Dis 2014;210:354-362.

22. Molina JM, Clotet B, van Lunzen J, et al:: Once-daily dolutegravir versus darunavir plus ritonavir for treatmentnaive adults with HIV-1 infection (FLAMINGO): 96 Week results from a randomised, open-label, phase $3 \mathrm{~b}$ study. Lancet HIV 2015;2:e127-e136. 
23. Orrell C, Hagins DP, Belonosova E, et al.: Fixed-dose combination dolutegravir, abacavir, and lamivudine versus ritonavir-boosted atazanavir plus tenofovir disoproxil fumarate and emtricitabine in previously untreated women with HIV-1 infection (ARIA): Week 48 results from a randomised, open-label, non-inferiority, phase $3 \mathrm{~b}$ study. Lancet HIV 2017;4:e536-e546.

24. Gallant J, Lazzarin A, Mills A, et al.: Bictegravir, emtricitabine, and tenofovir alafenamide versus dolutegravir, abacavir, and lamivudine for initial treatment of HIV-1 infection (GS-US-380-1489): A double-blind, multicentre, phase 3, randomised controlled non-inferiority trial. Lancet 2017;390:2063-2072.

25. Sax PE, Pozniak A, Montes ML, et al.: Coformulated bictegravir, emtricitabine, and tenofovir alafenamide versus dolutegravir with emtricitabine and tenofovir alafenamide, for initial treatment of HIV-1 infection (GSUS-380-1490): A randomised, double-blind, multicentre, phase 3, non-inferiority trial. Lancet 2017;390:20732082.

26. Arts EJ, Hazuda DJ: HIV-1 antiretroviral drug therapy. Cold Spring Harb Perspect Med 2012;2:a007161.

27. Hightower KE, Wang R, Deanda F, et al.: Dolutegravir (S/GSK1349572) exhibits significantly slower dissociation than raltegravir and elvitegravir from wild-type and integrase inhibitor-resistant HIV-1 integrase-DNA complexes. Antimicrob Agents Chemother 2011;55:45524559.

28. White K, Niedziela-Majka A, Novikov N, et al:: Bictegravir dissociation half-life from HIV-1 G140S/Q148H integrase-DNA complexes. Abstract presented at: Conference on Retroviruses and Opportunistic Infections; February 13-16, 2017. Seattle, WA.

29. La Porte C: Inhibitory quotient in HIV pharmacology. Curr Opin HIV AIDS 2008;3:283-287.

30. Tivicay [package insert]. ViiV Healthcare, Research Triangle Park, NC, 2018.

31. Kobayashi M, Yoshinaga T, Seki T, et al:: In vitro antiretroviral properties of S/GSK1349572, a next-generation HIV integrase inhibitor. Antimicrob Agents Chemother 2011;55:813-821.

32. Sampah ME, Shen L, Jilek BL, Siliciano RF: Doseresponse curve slope is a missing dimension in the analysis of HIV-1 drug resistance. Proc Natl Acad Sci U S A 2011; 108:7613-7618.

33. Min S, Song I, Borland J, et al.: Pharmacokinetic (PK) and pharmacodynamic (PD) relationship of S/GSK1349572, a next generation integrase inhibitor (INI), in HIV-1 infected patients. Abstract presented at: 5th IAS Conference on HIV Pathogenesis, Treatment and Prevention; July 19-22, 2009. Capetown, South Africa.

34. Min S, Sloan L, DeJesus E, et al.: Antiviral activity, safety, and pharmacokinetics/pharmacodynamics of dolutegravir as 10-day monotherapy in HIV-1-infected adults. AIDS 2011;25:1737-1745.

35. Osterberg LG, Urquhart J, Blaschke TF: Understanding forgiveness: Minding and mining the gaps between pharmacokinetics and therapeutics. Clin Pharmacol Ther 2010; $88: 457-459$.

36. O'Connor JL, Gardner EM, Esser S, et al.: A simple selfreported adherence tool as a predictor of viral rebound in people with viral suppression on antiretroviral therapy. HIV Med 2016;17:124-132.
37. Elliot E, Amara A, Jackson A, et al: Dolutegravir and elvitegravir plasma concentrations following cessation of drug intake. J Antimicrob Chemother 2016;71:1031-1036.

38. Genvoya [Gilead Sciences, Inc.]. Gilead Sciences, Inc., Foster City, CA, 2019.

39. Iwamoto M, Wenning LA, Petry AS, et al.: Safety, tolerability, and pharmacokinetics of raltegravir after single and multiple doses in healthy subjects. Clin Pharmacol Ther 2008;83:293-299.

40. Gallant JE, Thompson M, DeJesus E, et al: Antiviral activity, safety, and pharmacokinetics of bictegravir as 10day monotherapy in HIV-1-infected adults. J Acquir Immune Defic Syndr 2017;75:61-66.

41. Yuen GJ, Lou Y, Bumgarner NF, et al.: Equivalent steadystate pharmacokinetics of lamivudine in plasma and lamivudine triphosphate within cells following administration of lamivudine at 300 milligrams once daily and 150 milligrams twice daily. Antimicrob Agents Chemother 2004; 48:176-182.

42. Moore KH, Barrett JE, Shaw S, et al.: The pharmacokinetics of lamivudine phosphorylation in peripheral blood mononuclear cells from patients infected with HIV-1. AIDS 1999;13:2239-2250.

43. Shen L, Rabi SA, Siliciano RF: A novel method for determining the inhibitory potential of anti-HIV drugs. Trends Pharmacol Sci 2009;30:610-616.

44. Laskey SB, Siliciano RF: Quantitative evaluation of the antiretroviral efficacy of dolutegravir. JCI Insight 2016;1: e90033.

45. Wandeler G, Buzzi M, Anderegg N, et al.: Virologic failure and HIV drug resistance on simplified, dolutegravir-based maintenance therapy: Systematic review and meta-analysis. F1000Res 2018;7:1359.

46. Corado KC, Caplan MR, Daar ES: Two-drug regimens for treatment of naive HIV-1 infection and as maintenance therapy. Drug Des Devel Ther 2018;12:3731-3740.

47. Cahn P, Andrade-Villanueva J, Arribas JR, et al.: Dual therapy with lopinavir and ritonavir plus lamivudine versus triple therapy with lopinavir and ritonavir plus two nucleoside reverse transcriptase inhibitors in antiretroviraltherapy-naive adults with HIV-1 infection: 48 Week results of the randomised, open label, non-inferiority GARDEL trial. Lancet Infect Dis 2014;14:572-580.

48. Raffi F, Babiker AG, Richert L, et al.: Ritonavir-boosted darunavir combined with raltegravir or tenofoviremtricitabine in antiretroviral-naive adults infected with HIV-1: 96 Week results from the NEAT001/ANRS143 randomised non-inferiority trial. Lancet 2014;384:19421951.

49. Perez-Molina JA, Rubio R, Rivero A, et al.: Dual treatment with atazanavir-ritonavir plus lamivudine versus triple treatment with atazanavir-ritonavir plus two nucleos(t)ides in virologically stable patients with HIV-1 (SALT): 48 Week results from a randomised, open-label, noninferiority trial. Lancet Infect Dis 2015;15:775-784.

50. Reynes J, Trinh R, Pulido F, et al.: Lopinavir/ritonavir combined with raltegravir or tenofovir/emtricitabine in antiretroviral-naive subjects: 96-Week results of the PROGRESS study. AIDS Res Hum Retroviruses 2013;29:256-265.

51. Second-Line Study Group, Boyd MA, Kumarasamy N, et al:: Ritonavir-boosted lopinavir plus nucleoside or nucleotide reverse transcriptase inhibitors versus ritonavirboosted lopinavir plus raltegravir for treatment of HIV-1 
infection in adults with virological failure of a standard first-line ART regimen (SECOND-LINE): A randomised, open-label, non-inferiority study. Lancet 2013;381:20912099.

52. Walmsley S, Baumgarten A, Berenguer J, et al.: Brief report: Dolutegravir plus abacavir/lamivudine for the treatment of HIV-1 infection in antiretroviral therapy-naive patients: Week 96 and week 144 results from the SINGLE randomized clinical trial. J Acquir Immune Defic Syndr 2015;70:515-519.

53. Eron J, Hung C-C, Baril J-G, et al:: Initial viral load decline and response rates by baseline viral load strata with dolutegravir plus lamivudine versus dolutegravir plus teno- fovir disoproxil fumarate/emtricitabine: Pooled results from the GEMINI studies. Abstract presented at: HIV DART and Emerging Viruses; November 27-29, 2018. Miami, FL.

Address correspondence to: Romina Quercia ViiV Healthcare CN12, 980 Great West Road Brentford, Middlesex, TW8 9GS United Kingdom

E-mail: romina.p.quercia@viivhealthcare.com 